\title{
The effect of universal maternal antenatal iron supplementation on neurodevelopment in offspring: a systematic review and meta-analysis
}

\author{
C. Jayasinghe ${ }^{1 *}\left(\mathbb{D}\right.$, R. Polson ${ }^{2}$, H. C. van Woerden ${ }^{2}$ and P. Wilson ${ }^{3}$
}

\begin{abstract}
Background: Although antenatal iron supplementation is beneficial to mothers, its impact on the neurodevelopment of offspring is controversial. A systematic review and meta-analysis was undertaken to assess whether routine maternal antenatal iron supplementation confers later neurodevelopmental benefit to offspring.

Methods: Electronic databases were searched using MESH terms or key words and identified papers were reviewed by two independent reviewers. The study quality was assessed using the Cochrane risk of bias assessment tool. The review was registered in the PROSPERO CRD data base.

Results: Seven publications were identified, based on four randomised trials published between 2006 and 2016. Three of the trials were in the Asian sub-continent. A range of tools were used to evaluate neurodevelopment. Meta-analysis of outcomes from the three RCTs meeting our inclusion criteria showed minimal effect of antenatal iron supplementation on the neurodevelopment of offspring, which was not statistically significant: weighted mean difference of 0.54 (95\% Cl: -0.67 to 1.75 ); test for overall effect $Z=0.87 ; p=0.38$; and heterogeneity $48 \%$. Meta-analysis of outcomes of these $R C T s$ at later stages of development produced similar results.

Conclusions: The benefit of routine antenatal iron supplementation on neurodevelopment in offspring was not statistically significant in this relatively limited set of trials, and some benefit cannot be excluded in areas with a high prevalence of maternal anaemia. A large randomized controlled trial showing significant benefit would be required to modify our conclusions.
\end{abstract}

Keywords: Iron supplementation, Antenatal, Offspring, Neurodevelopment, Iron deficiency anaemia

\section{Background}

Iron supplementation during pregnancy is routinely provided in many countries. The WHO currently recommends that $30-60 \mathrm{mg}$ of elemental iron is given daily from as early as possible during pregnancy [1]. This public health policy aims to improve pregnancy outcomes and to reduce maternal anaemia [2, 3]. The beneficial effect of iron supplementation during the antenatal period in relation to birth weight, physical growth and perinatal mortality has been evaluated in several studies

\footnotetext{
* Correspondence: chinthaj@yahoo.com

${ }^{1}$ Ministry of Health, 385, Ven. Baddegama Wimalawansa Thero Mawatha,

Colombo 10, Sri Lanka

Full list of author information is available at the end of the article
}

$[2,4]$. An association has been shown between poor performance in mental and psychomotor tests and lower iron status in utero [5]. A randomized trial conducted in Bangladesh supplementing iron in various doses, and providing multiple micronutrients in food items for antenatal mothers, did not shown any significant effect on motor development of offspring at seven months old [6]. However, a finding of the northern Finnish birth cohort of 1966 was that low maternal haemoglobin levels in the final stage of pregnancy was linked to poorer educational achievement of offspring [7]. Another study showed that antenatal iron and folic acid supplementation reduced the prevalence of anaemia from $67 \%$ to 38 . $4 \%$, and that there was an inverted $U$ shaped relationship

(c) The Author(s). 2018 Open Access This article is distributed under the terms of the Creative Commons Attribution 4.0 International License (http://creativecommons.org/licenses/by/4.0/), which permits unrestricted use, distribution, and reproduction in any medium, provided you give appropriate credit to the original author(s) and the source, provide a link to the Creative Commons license, and indicate if changes were made. The Creative Commons Public Domain Dedication waiver (http://creativecommons.org/publicdomain/zero/1.0/) applies to the data made available in this article, unless otherwise stated. 
between maternal haemoglobin concentration and motor function of one year old children [8]. Antenatal supplementation with multiple micro-nutrients including iron and folic acid has been shown to improve motor and cognitive function of offspring at 3.5 years more effectively than supplementation with iron and folic acid alone [9]. These studies give rise to the question as to whether antenatal iron supplementation on its own leads to better neurodevelopment in offspring, which is the focus of this review.

The uncertainty in current evidence has been recognised by previous researchers who have recommended that the long term effects on child development of iron supplementation during pregnancy should be assessed as an area of priority [10-12]. The last available review of the effect of iron supplementation in non-anaemic pregnant women, infants and young children on mental performance and psychomotor development of children was only able to include one randomised controlled trial (RCT), which was undertaken in Australia and was inconclusive [13]. We have therefore undertaken a systematic review of the literature to examine the effect of iron supplementation in pregnancy on neurodevelopment in offspring. Neurodevelopment has been defined in this context to include aspects of behaviour, cognitive development, mental development or intellectual development.

\section{Methods}

\section{Search strategy}

Electronic databases, namely Ovid Medline, EMBASE, CINAHL, SCOPUS, Cochrane Central Register of Controlled Trials (CENTRAL), and Web of Science were searched between 5th January and 10th February 2016. The review included randomised controlled trials and cohort studies reporting on the routine use of iron supplementation during pregnancy and associated assessment of child development as an outcome. The inclusion of cohort studies is in line with best practice where published evidence is very sparse [14]. Non-randomized observational studies were not included to minimize the risk of selection bias and confounding (we found no such studies in our literature searches in any case). Both medical subject headings and free text keywords were utilised as search terms. In this review, synonyms for the term 'child development' included 'children's brain development,' 'IQ,' 'cognitive, psychomotor or behavioural development' (See search criteria in the Additional file 1). Although we planned to include cohort studies and of iron supplementation in pregnancy followed by assessment of child development, in addition to RCTs, none fulfilled the inclusion criteria.

Papers reporting the outcome of interest using any type of neurodevelopmental assessment tools were accepted for the review. The references in the identified articles and in related review articles were manually searched for additional literature that might otherwise have been missed by the electronic databases. The review included only original research articles published as full text in the English language and conducted on human beings.

\section{Inclusion criteria}

The inclusion criteria were: RCTs and cohort studies, reporting the association between iron supplementation during pregnancy and neurodevelopment in offspring undertake before the offspring had reached puberty, in practice, 12 years of age. Iron supplementation had to be the key difference between at least one intervention group and one control group.

PICO $[15,16]$ definitions:

Population: Pregnant women

Intervention: Iron supplementation (or iron with a micronutrient, where the comparator was the micronutrient alone)

Comparator: Placebo supplementation, or no supplementation, or micronutrient supplementation but without iron or ferrous compounds

Outcome: Child neurodevelopment, including mental development, cognitive function, psychomotor development, IQ, behaviour or other aspects of neurodevelopment

\section{Study selection}

Titles and abstracts retrieved from the search were jointly assessed by two reviewers (JC \& $\mathrm{HvW}$ ) for eligibility by reading titles and, where appropriate, abstracts. Studies that were obviously irrelevant were excluded. A study selection flow diagram is shown in Fig. 1 based on the PRISMA tool [17]. The full text of potentially relevant remaining articles was assessed by one reviewer, with review by a second person, to establish final eligibility. An update of the literature search was carried out to 30th July 2016. One study published in May 2016 was found and was included in the review [18].

\section{Data extraction and quality review process}

Data from the included studies are summarized in Table 1. A data extraction instrument designed in line with Cochrane data extraction sheets was used [19], which included the author, title, journal, year of publication, study setting, study design, study population, sample size, participants' characteristics, country where the research was carried out, type of iron supplementation, definition of iron deficiency anaemia, the method or tool used to assess child development, follow up period and study outcomes relevant to the review. Key data from each of the selected studies were at first reviewed independently by two reviewers (JC \& HvW) and then jointly 


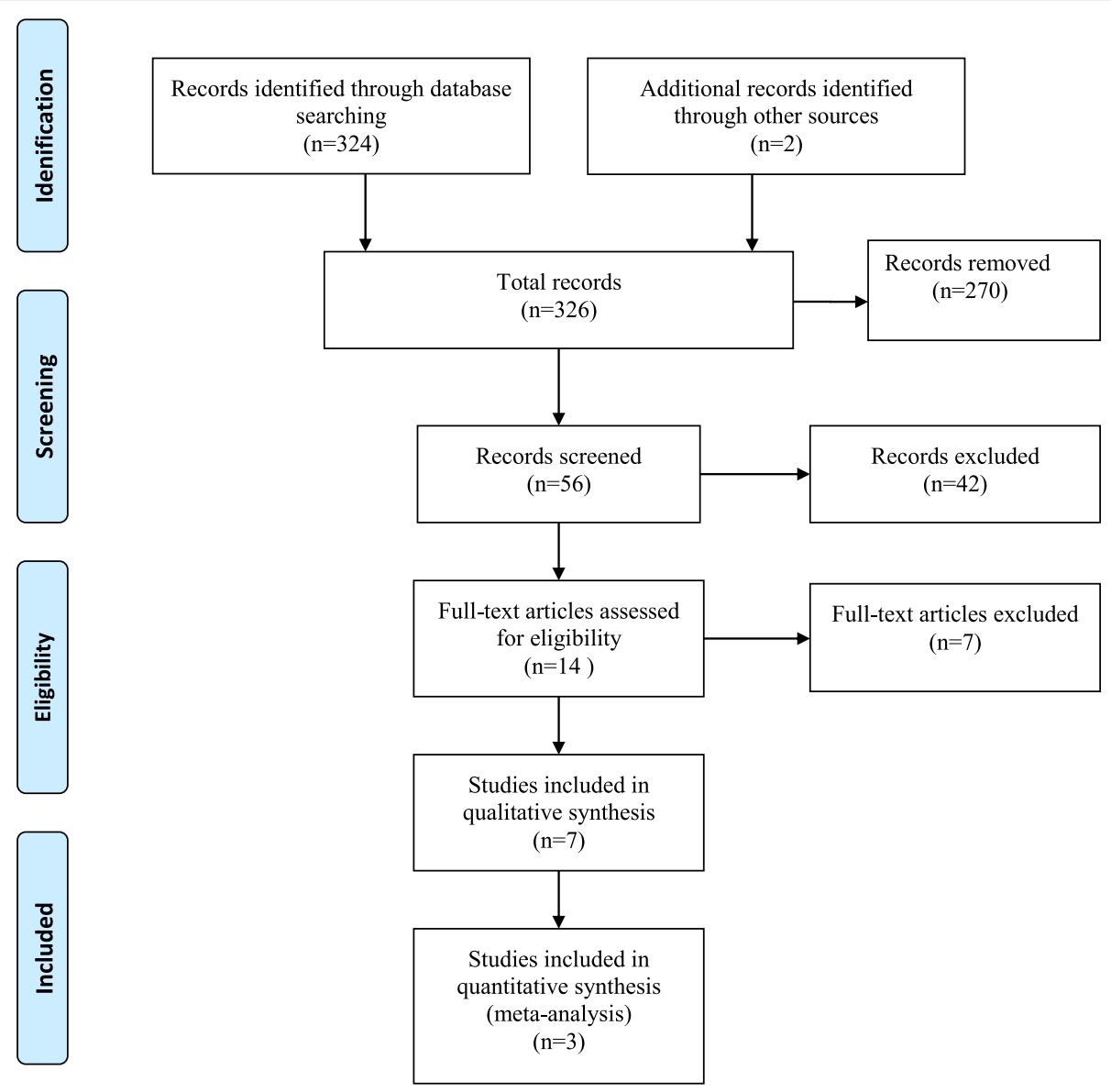

Fig. 1 PRISMA Flow diagram for identified studies

for quality assessment using the Cochrane Risk of Bias check list [2]. Discrepancies were forwarded for arbitration to the third reviewer (PW). We had only two disagreements regarding the blindness of intervention group of the participants in Parsons et el. [20] and inclusion of a Mendelian randomization study for the review [21]. Risk of bias was rated as high, low or unclear (see Fig. 2).

\section{Analysis}

A meta-analysis was undertaken using Review Manager 5.3 [22]. Studies were excluded from the meta-analysis if they included the same participants who had been reported in another included paper. Where several studies followed up the same group of participants, the study entered into the meta-analysis was that with the length of follow up that was closest to other included studies. Forest plots were created using Review Manager and weighted mean differences were calculated.

A random effects model was used, as there was a potential for heterogeneity arising out of the range of assessment methods used by the included studies. Under such circumstances this is more robust than using a fixed effects model. The review was registered in the PROSPERO CRD data base, registration number CRD 42016037114.

\section{Results}

A total of 324 articles were identified by searching electronic databases and a further two were identified by manual bibliographic searches (Fig. 1). Of those, 270 articles were excluded during initial screening because they did not report the effect of iron supplementation on child neurodevelopment. The full texts of the remaining 56 articles were again reviewed to assess whether their research objectives met our inclusion criteria. This full text review led to exclusion of a further 42 articles as the outcome of interest was outside the scope of our objectives. Hence, 15 articles were selected by both reviewers, one of which was excluded because the intervention included both iron and folic acid [12]. Six articles were excluded as they assessed different doses of antenatal iron but did not utilise a control group who did not receive a supplement [6-9, 23, 24]. One Mendelian randomization study was removed because final analysis was undertaken according to the 


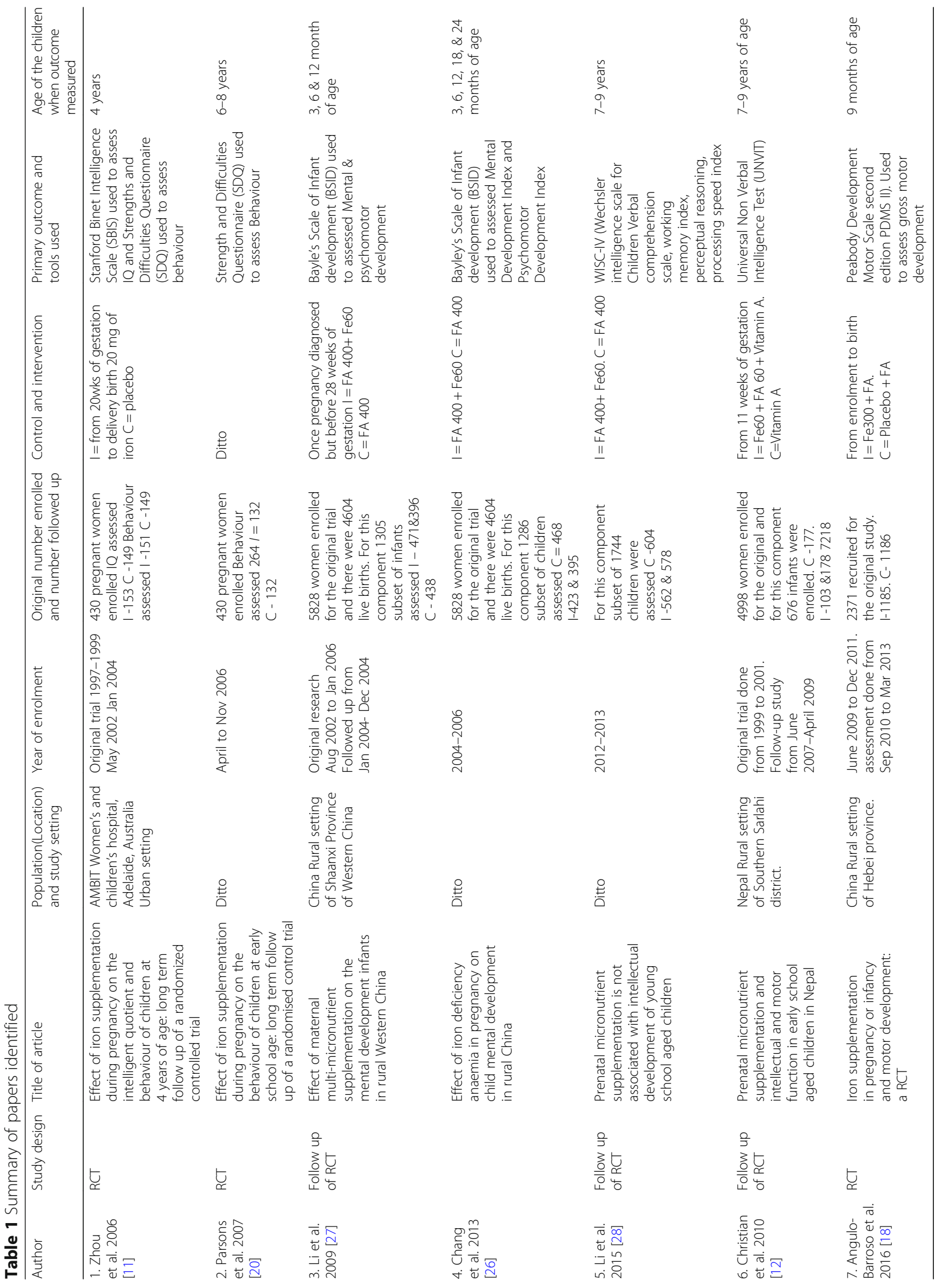




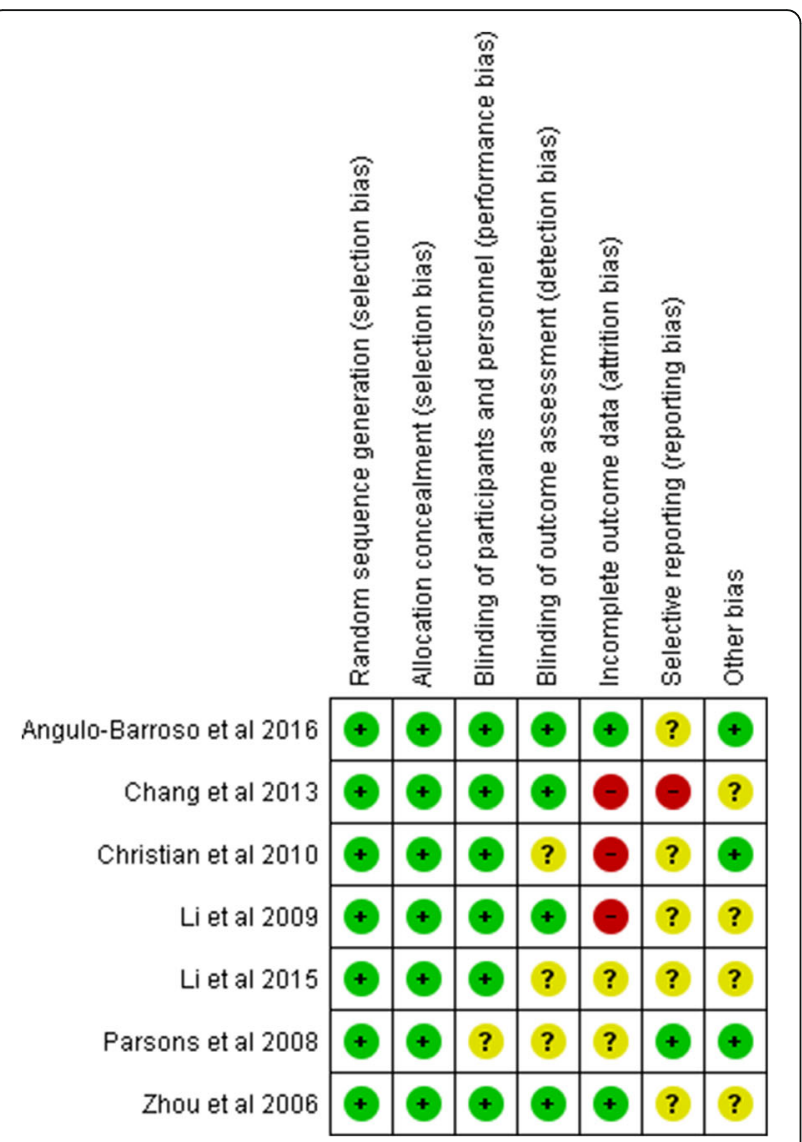

Fig. 2 Risk of Bias Assessment

genetic variant and the effect of iron supplementation or non-supplementation could not be established [21]. The remaining seven articles were included in the qualitative synthesis (Fig. 1).

\section{Study characteristics}

The seven papers we reviewed were based on four trials and were published between 2006 and 2016 (Table 1). Six papers were extensions of three original RCTs. Sample size varied from 264 to 1714 . The reviewed papers are summarised in Table 2 and in the Supplementary file. They included pregnant women from three different countries: China, Australia and Nepal.

There was variation in the dose of iron supplementation and the gestation period at which supplementation was initiated. Maternal iron supplementation varied from $20 \mathrm{mg}$ daily up to $300 \mathrm{mg}$ daily (Table 1 ). Initiation of iron supplementation varied from 11 weeks gestation to 20 weeks gestation. Two RCTs were carried out in areas where anaemia prevalence was high and one in an area where it was low $[11,20,25]$.

Three publications [26-28] were follow up studies of a single RCT [29], with outcomes measured at different
Table 2 Summary of parameters for the intervention and control groups for the key studies

\begin{tabular}{lllllllll}
\hline Authors & \multicolumn{3}{l}{ Intervention group } & & \multicolumn{3}{c}{ Control group } \\
\cline { 2 - 3 } & Mean & SD & N & & Mean & SD & N \\
\hline Zhou et al. 2006 [11] & 109 & 11 & 153 & & 109 & 11 & 149 \\
Parsons et al. 2008 [20] & 8.6 & 6.3 & 132 & 9.2 & 5.9 & 132 \\
Li et al. 2009 [27] & 102.44 & 4.475 & 438 & 102.65 & 4.42 & 471 \\
Chang et al. 2013 [26] & 100.2 & 15.7 & 466 & 98 & 15.3 & 384 \\
Li et al. 2015 [28] & 88.98 & 12.69 & 562 & 89.82 & 14.07 & 604 \\
Christian et al. 2010 [12] & 51.7 & 8.5 & 103 & 48.2 & 10.2 & 177 \\
Angulo Barroso et al. 2016 [18] & 87.5 & 0.95 & 298 & 87.4 & 0.95 & 288 \\
\hline
\end{tabular}

stages in the development in the offspring, where mothers had received iron supplementation. Similarly, another two publications $[11,20]$ were based on another RCT where mothers had received iron supplementation, with outcomes measured at different stages in the development in the offspring [25]. We excluded the study carried out in Nepal for the meta-analysis [12]. This was because in the original study there was a control group receiving folic acid only supplementation, but this group was not included in the follow up study [30]. In the trial by Angulo Barroso et al., although there were four arms, for this review we only considered the outcomes related to the arm that received iron supplementation during pregnancy and the control group [18]. The children who received iron supplementation after birth were not included in our analysis.

\section{Anaemia prevalence}

The prevalence of anaemia varied markedly across the countries included in this review. A study linked to the RCT in China gave a prevalence of anaemia undertaken in the third trimester of gestation of 57\% [29]. In Nepal the baseline prevalence of anaemia in the study area was more than $40 \%$ [30, 31]. In contrast the prevalence of iron deficiency anaemia among those recruited in Australia was 11\% [25]. The estimated global prevalence of anaemia in the general population is $24.8 \%$, with an estimated anaemia prevalence in preschool children of $47.4 \%$, in pregnant women $41.8 \%$ and in non-pregnant women $30.2 \%$ [32].

\section{Quality of reporting}

The quality of each of the studies was assessed according to the Cochrane Risk of Bias tool. All the papers were generally acceptable for most items in the quality assessment matrix [19]. All studies used random sequence generation and allocation concealment. In all, except one paper [20] participants were blinded. The extent of blinding of outcome assessment was unclear in three studies $[12,20,28]$. There was very high loss to follow 
up in three papers $[12,26,27]$ and follow up rates were unclear in two papers (Fig. 2). Analysis of primary outcome data was undertaken on an 'intention to treat' basis in all except one study [26].

\section{Mental development outcome}

Different scales of measurement were used in different studies, namely: Bayley's Scale of Infant Development (BSID); Stanford-Binet Intelligence Scale (SBIS); Strengths and Difficulties Questionnaire (SDQ); Wechsler Intelligence Scale for Children (WISC); Universal Nonverbal Intelligence Test (UNVIT) and the Peabody Developmental Motor Scale (PDMS). Although not initially designed for that purpose, the SDQ has been used for studies to assess language, social, emotional problems and as a neurodevelopment assessment tool at younger ages, and was therefore considered a valid tool [33]. All the other tools have been used to assess intelligence and cognitive function in children [34].

\section{Effect of intervention}

Three publications were not included in the metaanalysis as they used the same participants at different ages [20, 27, 28]. The RCT carried out in Nepal [12] was not included as the intervention group was supplemented with both iron and folic acid. Three RCTs were included in the meta-analysis and provided a weighted mean difference of 0.54 units ( $95 \%$ CI -0.67 to 1.75 ), with an overall effect size $\mathrm{z}=0.87(p=0.38)$ and a heterogeneity of $48 \%(p=0.15)$. The level of heterogeneity was moderate $\left(\mathrm{I}^{2}=48 \%\right)$ and not statistically significant (Fig. 3). The results suggest that routine (as opposed to targeted) maternal iron supplementation does not have a statistically significant effect on later neurodevelopment in offspring.

\section{Discussion}

The primary objective of this systematic review was to determine whether antenatal iron supplementation provided any neurodevelopment benefit for offspring. Among the seven eligible publications reviewed, only one study was found that primarily focused on iron supplementation in pregnancy and motor development of offspring [18]. The other six publications were based on
RCTs where the original focus was on different objectives and extensions of the initial trials were undertaken to examine child neurodevelopmental effects. Our conclusion is that any effect of routine iron supplementation on its own is likely to be very small.

We would suggest that the benefits of routine antenatal supplementation are likely to be related to the underlying prevalence of anaemia in a given population. The greatest effect was observed where anaemia prevalence was highest [26] and the least effect was observed where anaemia prevalence was lowest [11].

There is evidence that the global prevalence of anaemia in pregnancy is falling, as a study carried out to estimate the global mean haemoglobin concentration among women of reproductive age (15-49 years) has shown that anaemia prevalence decreased from $43 \%$ to $38 \%$ in pregnant women from 1995 to 2011 [35]. This study suggests that in countries where the prevalence of anaemia is low or falling, there may be a case for limiting antenatal prescription of iron to women who are anaemic and have low iron stores.

An outstanding question, which merits further research, is whether iron supplementation is best seen as one of a cluster of micronutrients that need to be available during foetal growth to maximise neurodevelopment, as two studies have provided some evidence to support antenatal iron supplementation when provided in conjunction with other micronutrients. In one study carried out in Nepal, iron and folic acid supplementation improved mean Universal Nonverbal Intelligence Test scores on psychometric testing compared to a control group [12]. A study in China also demonstrated a small improvement in mental development raw scores at one year of age in an iron supplementation group, compared to a non-supplementation group [26]. Another study showed a lower mental development index in children born to mothers with iron deficiency anaemia during pregnancy compared to those whose mothers were not iron deficient during pregnancy [27]. As previously stated, this may reflect the interplay between the availability of iron and other aetiological factors.

Although not include it in the meta analysis, a study carried out in rural Vietnam demonstrated significant

\begin{tabular}{|c|c|c|c|c|c|c|c|c|c|c|}
\hline \multirow[b]{2}{*}{ Study or Subgroup } & \multicolumn{3}{|c|}{ Iron supplementation } & \multicolumn{3}{|c|}{ Control } & \multirow[b]{2}{*}{ Weight } & \multirow{2}{*}{$\begin{array}{l}\text { Mean Difference } \\
\text { IV, Random, } 95 \% \mathrm{Cl}\end{array}$} & \multirow{2}{*}{\multicolumn{2}{|c|}{$\begin{array}{c}\text { Mean Difference } \\
\text { IV, Random, } 95 \% \mathrm{Cl}\end{array}$}} \\
\hline & Mean & SD & Total & Mean & SD & Total & & & & \\
\hline Angulo-Barroso et al 2016 & 87.5 & 0.95 & 298 & 87.4 & 0.95 & 288 & $61.0 \%$ & $0.10[-0.05,0.25]$ & -1] & \\
\hline Chang et al 2013 & 100.2 & 15.7 & 466 & 98 & 15.3 & 384 & $21.8 \%$ & $2.20[0.11,4.29]$ & & \\
\hline Zhou et al 2006 & 109 & 11 & 153 & 109 & 11 & 149 & $17.2 \%$ & $0.00[-2.48,2.48]$ & & \\
\hline Total $(95 \% \mathrm{Cl})$ & & & 917 & & & 821 & $100.0 \%$ & $0.54[-0.67,1.75]$ & & \\
\hline \multicolumn{6}{|c|}{$\begin{array}{l}\text { Heterogeneity: } \text { Tau }^{2}=0.62 ; \mathrm{Chi}^{2}=3.86, \mathrm{df}=2(P=0.15) ;\left.\right|^{2}=48 \% \\
\text { Test for overall effect: } Z=0.87(P=0.38)\end{array}$} & & & & $\begin{array}{cccc}-4 & -2 & 0 & 1 \\
\text { Iron supplementation } & \text { Control }\end{array}$ & 4 \\
\hline
\end{tabular}


improvement in infant cognitive outcomes at six months of age following twice weekly antenatal iron supplements rather than daily supplements [36]. In an Indonesian trial, supplementation with daily iron, weekly iron with Vitamin A, and weekly iron alone was provided from 18 weeks of gestation until delivery followed by assessment of cognitive and psychomotor development of infants. The infants of all three supplemented groups had similar mental and psychomotor development indices at six and twelve months of age [25].

It is important to consider whether routine iron supplementation could have adverse, as well as beneficial effects, as the possibility exists of adverse effects from excessive iron supplementation in those who are not iron deficient. Abnormal teacher rated peer relationship problem subscale scores in the Strengths and Difficulties Questionnaire were associated with iron supplementation in an Australian study ( $R R=3.70 ; 95 \%$ CI 1.06 to $12.91 ; p=0.026)$ [20]. In this study, the attrition rate was almost 50\% (only 112 out of 216 who were initially randomized completed the study) and the results may have been biased by differential drop out of healthy children. The findings of this study therefore need interpreted with caution. In summary, the meta-analysis of three RCTs did not show a statistically significant benefit from routine antenatal iron supplementation on the neurodevelopment of offspring: weighted mean difference of 0 . 54 units (95\% CI 0.67 to 1.75$)$.

\section{Strengths and limitations}

This review included RCTs without limiting the date of publication. It provides a substantial update on a previous review, which had slightly different aims, but only identified a single RCT [13]. The assessment of quality in the RCTs was based on the methods described in the original trials, bearing in mind that the original studies were conducted with different objectives in some cases. The tools used to assess the mental development of children varied widely, as did the neurodevelopmental domains that were evaluated, and some tools had not been validated in the language into which they had been translated.

A limitation of our study is that we may have missed unpublished data or studies published in the grey literature. We may also have missed studies that were not published in English. The majority of the included trials were performed in Far Eastern countries and the generalizability to other areas in the world requires some caution.

The most appropriate follow up period for a study such as this is difficult to determine. Assessment a week after birth would clearly provide an inadequate follow up period and follow up after the age of 12 years is probably affected by a wide range of other environmental factors including puberty. Follow up at younger ages might reduce heterogeneity, as most studies undertake follow up within the first five years. However, follow up at older ages is probably a better proxy for identifying lifelong impacts of antenatal iron supplementation. A case can be made for either approach. Sensitivity analysis around age (not shown) did not indicate significant differences in the outcome of the meta-analysis when the age at which assessment was undertaken was varied. In general, neurodevelopmental deficits present in early life will be continued to some extent in later years so the direction of any effects should be the same regardless of age or precise developmental assessment method.

Assessment of neurodevelopmental function is challenging. There is no universally recognized method of assessing such development, and as a result different studies inevitably use different tools. Stanford Binet Intelligence Scale is used to assess cognitive ability and intelligence, Bayley's Scale of Infant Development is used for cognitive, language, motor, social and emotional assessment and the Peabody development scale is used for fine and gross motor development assessment. Restricting our analysis to only one dimension of neuro-development such as cognitive function might have missed wider effects, but does introduce conceptual heterogeneity.

It could be argued that this review should be restricted to a descriptive analysis of the papers that have been identified, on the basis of the limited and diverse nature of the assessment methods that were used. However, quantification of results does add value, even when this needs to be presented with appropriate caveats. On balance, we believe that the advantages of including a range of studies in our meta-analysis outweigh potential disadvantages and a meta-analysis has therefore been provided.

\section{Implication for research}

It is possible to speculate that one micronutrient deficiency is often associated with the presence of other micronutrient deficiencies. As a result, future research might assess whether larger benefits can be obtained by the use of enriched diets with high multi-micronutrient content, or multi-micronutrient supplementation, which would address deficits across a wider range of enzymatic pathways and potentially have a greater overall effect on neurodevelopment in the foetal brain.

\section{Conclusion}

We have attempted to establish whether there are positive neurodevelopmental outcomes from antenatal iron supplementation and our conclusion is that there was little or no evidence of benefit in routine use. Any effect appears to be small. We consider that a large randomized controlled trial of universal iron supplementation would be required to substantially change our conclusions. 


\section{Additional file}

Additional file 1: Part 1: Demonstration of search strategy for Medline; Part 2: Summary of the selected studies. (DOCX $24 \mathrm{~kb}$ )

\section{Abbreviations}

BSID: Bayley's Scale of Infant Development; IQ: Intelligence Quotient; MESH: Medical Subject Headings; PDMS: Peabody Developmental Motor Scale; PICO: Population, Intervention, Comparator, Outcome;

PRISMA: Preferred Reporting Items for Systematic Reviews and MetaAnalyses; RCT: Randomised controlled trial; SBIS: Stanford-Binet Intelligence Scale; SDQ: Strengths and Difficulties Questionnaire; UNVIT: Universal Nonverbal Intelligence Test; WISC: Wechsler Intelligence Scale for Children

\section{Acknowledgements}

The authors would like to acknowledge statistical advice from staff at the University of Aberdeen.

\section{Funding}

One author (CJ) was funded by the Ministry of Health, Government of Sri Lanka.

\section{Availability of data and materials}

Supporting data can be obtained from the corresponding author

\section{Authors' contributions}

The conception and design of the study was provided by PW; acquisition of papers, analysis and interpretation were led by $\mathrm{CJ}, \mathrm{RP}$ and $\mathrm{HvW}$ and was checked by PW; the manuscript was first drafted by CJ and HvW; all the authors were involved in reviewing the draft. All authors have read and approved the final manuscript.

\section{Ethics approval and consent to participate}

Not applicable.

\section{Competing interests}

The authors declare that they have no competing interests.

\section{Publisher's Note}

Springer Nature remains neutral with regard to jurisdictional claims in published maps and institutional affiliations.

\section{Author details}

${ }^{1}$ Ministry of Health, 385, Ven. Baddegama Wimalawansa Thero Mawatha, Colombo 10, Sri Lanka. ${ }^{2}$ Centre for Health Science, University of the Highland and Islands, Old Perth Road, Inverness IV2 3JH, UK. ${ }^{3}$ Centre for Rural Health, University of Aberdeen, Old Perth Road, Inverness IV2 3JH, UK.

\section{Received: 30 November 2016 Accepted: 18 April 2018}

Published online: 04 May 2018

\section{References}

1. World Health Organization. Daily iron and folic acid supplementation in pregnant women. Geneva: WHO; 2012.

2. Peña-Rosas JP, De-Regil LM, Dowswell T, Viteri FE. Daily oral iron supplementation during pregnancy. The Cochrane library. New Jersey: John Wiley \& Sons; 2012.

3. Vucic V, Berti C, Vollhardt C, Fekete K, Cetin I, Koletzko B, Gurinovic M, van't Veer $P$. Effect of iron intervention on growth during gestation, infancy, childhood, and adolescence: a systematic review with meta-analysis. Nutr Rev. 2013;71(6):386-401.

4. Imdad A, Bhutta ZA. Routine iron/folate supplementation during pregnancy: effect on maternal anaemia and birth outcomes. Paediatr Perinat Epidemiol. 2012;26(s1):168-77.

5. Tamura T, Goldenberg RL, Hou J, Johnston KE, Cliver SP, Ramey SL, Nelson KG. Cord serum ferritin concentrations and mental and psychomotor development of children at five years of age. J Pediatr. 2002;140(2):165-70.

6. Tofail F, Persson LÅ, El Arifeen S, Hamadani JD, Mehrin F, Ridout D, Ekström E-C, Huda SN, Grantham-McGregor SM. Effects of prenatal food and micronutrient supplementation on infant development: a randomized trial from the maternal and infant nutrition interventions, Matlab (MINIMat) study. Am J Clin Nutr. 2008:87(3):704-11.

7. Fararouei M, Robertson C, Whittaker J, Sovio U, Ruokonen A, Pouta A, Hartikainen A-L, Jarvelin M-R, Hyppönen E. Maternal $\mathrm{Hb}$ during pregnancy and offspring's educational achievement: a prospective cohort study over 30 years. Br J Nutr. 2010;104(9):1363-8.

8. Mireku MO, Davidson LL, Koura GK, Ouedraogo S, Boivin MJ, Xiong X, Accrombessi MMK, Massougbodji A, Cot M, Bodeau-Livinec F. Prenata hemoglobin levels and early cognitive and motor functions of one-year-old children. Pediatrics. 2015;136:e76-83.

9. Prado EL, Alcock KJ, Muadz H, Ullman MT, Shankar AH. Maternal multiple micronutrient supplements and child cognition: a randomized trial in Indonesia. Pediatrics. 2012;130:e536-46.

10. Grantham-McGregor S, Ani C. A review of studies on the effect of iron deficiency on cognitive development in children. J Nutr. 2001;131(2): 649S-68S.

11. Zhou SJ, Gibson RA, Crowther CA, Baghurst P, Makrides M. Effect of iron supplementation during pregnancy on the intelligence quotient and behavior of children at 4 y of age: long-term follow-up of a randomized controlled trial. Am J Clin Nutr. 2006:83:1112-7.

12. Christian P, Murray-Kolb LE, Khatry SK, Katz J, Schaefer BA, Cole PM, LeClerq SC, Tielsch JM. Prenatal micronutrient supplementation and intellectual and motor function in early school-aged children in Nepal. JAMA. 2010;304:2716-23.

13. Szajewska H, Ruszczynski M, Chmielewska A. Effects of iron supplementation in nonanemic pregnant women, infants, and young children on the mental performance and psychomotor development of children: a systematic review of randomized controlled trials. Am J Clin Nutr. 2010;91:1684-90.

14. Peinemann F, Tushabe DA, Kleijnen J. Using multiple types of studies in systematic reviews of health care interventions-a systematic review. PLoS One. 2013;8(12):e85035.

15. Shamseer L, Moher D, Clarke $M$, Ghersi $D$, Liberati A, Petticrew $M$, Shekelle $P$, Stewart LA. Preferred reporting items for systematic review and meta-analysis protocols (PRISMA-P) 2015: elaboration and explanation. BMJ. 2015;349:g7647.

16. University of York Centre for Reviews and Dissemination. Systematic reviews: CRD's guidance for undertaking reviews in health care. York: University of York; 2009.

17. Moher D, Shamseer L, Clarke M, Ghersi D, Liberati A, Petticrew M, Shekelle P, Stewart LA. Preferred reporting items for systematic review and metaanalysis protocols (PRISMA-P) 2015 statement. In: Systematic Reviews. Volume 4, edn: Cochrance Collaboration, vol. 2015. p. 1.

18. Angulo-Barroso RM, Li M, Santos DCC, Bian Y, Sturza J, Jiang Y, Kaciroti N, Richards B, Lozoff B. Iron supplementation in pregnancy or infancy and motor development: a randomized controlled trial. Pediatrics. 2016;137: e20153547.

19. Higgins J, Green S. Cochrane handbook for systematic reviews of interventions version 5.1.0. New Jersey: John Wiley \& Sons; 2011.

20. Parsons AG, Zhou SJ, Spurrier NJ, Makrides M. Effect of iron supplementation during pregnancy on the behaviour of children at early school age: long-term follow-up of a randomised controlled trial. Br J Nutr. 2008;99:1133-9.

21. Lewis SJ, Bonilla C, Brion MJ, Lawlor DA, Gunnell D, Ben-Shlomo Y, Ness A, Smith GD. Maternal iron levels early in pregnancy are not associated with offspring IQ score at age 8, findings from a Mendelian randomization study. Eur J Clin Nutr. 2014;68:496-502.

22. Cochrane Collaboration. Review manager (RevMan) [computer program]. Version 5.3. Copenhagen: The Nordic Cochrane Collaboration Centre; 2012.

23. Schmidt MK, Muslimatun S, West CE, Schultink W, Hautvast JGAJ. Mental and psychomotor development in Indonesian infants of mothers supplemented with vitamin a in addition to iron during pregnancy. $\mathrm{Br} J$ Nutr. 2004:91:279.

24. Hanieh S, Ha TT, Simpson JA, Casey GJ, Khuong NC, Thoang DD, Thuy TT, Pasricha SR, Tran TD, Tuan T, et al. The effect of intermittent antenatal Iron supplementation on maternal and infant outcomes in rural Viet Nam: a cluster randomised trial. PLOS Med. 2013:10:e1001470.

25. Makrides M, Crowther CA, Gibson RA, Gibson RS, Skeaff CM. Efficacy and tolerability of low-dose iron supplements during pregnancy: a randomized controlled trial. Am J Clin Nutr. 2003;78:145-53. 
26. Chang S, Zeng L, Brouwer ID, Kok FJ, Yan H. Effect of Iron deficiency Anemia in pregnancy on child mental development in rural China. Pediatrics. 2013;131:e755-63.

27. Li Q, Yan H, Zeng L, Cheng Y, Liang W, Dang S, Wang Q, Tsuji I. Effects of maternal multimicronutrient supplementation on the mental development of infants in rural western China: follow-up evaluation of a double-blind, randomized, controlled trial. Pediatrics. 2009;123:e685-92.

28. Li C, Zeng L, Wang D, Yang W, Dang S, Zhou J, Yan H. Prenatal micronutrient supplementation is not associated with intellectual development of young school-aged children. J Nutr. 2015;145:1844-9.

29. Zeng L, Dibley MJ, Cheng Y, Dang S, Chang S, Kong L, Yan H. Impact of micronutrient supplementation during pregnancy on birth weight, duration of gestation, and perinatal mortality in rural western China: double blind cluster randomised controlled trial. BMJ. 2008;337:a2001.

30. Christian P. Effects of alternative maternal micronutrient supplements on low birth weight in rural Nepal: double blind randomised community trial. BMJ. 2003;326:571.

31. Huang X, Qu X, Yan W, Huang Y, Cai M, Hu B, Wu L, Lin H, Chen Z, Zhu C, et al. Iron deficiency anaemia can be improved after eradication of helicobacter pylori. Postgrad Med J. 2010;86:272-8.

32. McLean E, Cogswell M, Egli I, Wojdyla D, de Benoist B. Worldwide prevalence of anaemia, WHO vitamin and mineral nutrition information system, 1993-2005. Public Health Nutr. 2009;12:444-54.

33. Sim F, O'Dowd J, Thompson L, Law J, Macmillan S, Affleck M, Gillberg C, Wilson P. Language and social/emotional problems identified at a universal developmental assessment at 30 months. BMC Pediatr. 2013:13:206.

34. Hughes D, Bryan J. The assessment of cognitive performance in children: considerations for detecting nutritional in uences. Nutr Rev. 2003;61:413-22.

35. Mason J, Martorell R, Saldanha L, Shrimpton R. Reduction of anaemia. Lancet Glob Health. 2013;1(1):e4-6.

36. Tran TD, Tran T, Simpson JA, Tran HT, Nguyen TT, Hanieh S, Dwyer T, Biggs BA, Fisher J. Infant motor development in rural Vietnam and intrauterine exposures to anaemia, iron deficiency and common mental disorders: a prospective community-based study. BMC Pregnancy and Childbirth. 2014;14:8.

\section{Ready to submit your research? Choose BMC and benefit from}

- fast, convenient online submission

- thorough peer review by experienced researchers in your field

- rapid publication on acceptance

- support for research data, including large and complex data types

- gold Open Access which fosters wider collaboration and increased citations - maximum visibility for your research: over $100 \mathrm{M}$ website views per year

At BMC, research is always in progress.

Learn more biomedcentral.com/submissions 